\title{
New Electronic Memory Device Concepts Based on Metal Oxide-Polymer Nanostructures Planer Diodes
}

\author{
Asal Kiazadeh, Paulo R.F. Rocha, Qian Chen, and Henrique L. Gomes \\ Center of Electronic, Optoelectronic and Telecommunications, Faculdade de Ciências e Tecno- \\ logia, Universidade do Algarve, Campus de Gambelas, \\ 8005-139 Faro, Portugal
}

\begin{abstract}
Planar diodes fabricated with nano-structured silver oxide thin film show resistive switching effects. These structures can be programmed by voltage into two distinct resistive states and they are promising candidates for resistive random access memory (RRAM) devices. Cycle endurance on/off ration and charge retention are high. This wok provides insight into the mechanisms leading to the memory effects and to charge transport in these memory structures. Temperature dependent measurements show that charge carrier transport has activation energy of $70 \mathrm{meV}$ suggesting that tunneling is the operating mechanism in both resistive states. The change in resistance is caused by a large increase in charge carrier density. This finding is explained by assuming that carrier transport takes place through a percolative network of micro-conducting paths.
\end{abstract}

Keywords: Nanostructured Materials, Resistive Switching.

\section{Introduction}

Metal-oxide nanostructured material in a polymeric matrix exhibits different resistivity states. In the previous paper it has shown that the resistive switching will occur through the polymer capped nanostructure silver oxide embedded in a planer diode with two gold electrodes [1]. There are lots of reports showing different mechanisms for resistive switching observation [2-9].

Up to now, all switching devices prepared with nanoparticles have some functional components in common: (i) Need a polymer network with the nanostructure material which can be metal sulfide, oxide or halide (ii) The initial state of device before switching is a Pristine-state. The pristine device usually needs an Electroforming process. The electroforming process is voltage application sweeping in order to reach to soft breakdown of oxide or halide, the electric field needs to switch for each device is different due to various matrixes chemically in material, work function and physically such as difference in layer thickness. After an electroforming the reproducible on/off system often obtains with a voltage pulse at low resistance state (LRS) voltage region or high resistance (HRS) voltage domain. Lack of an organic matrix, a destructive electrical switching is observed. In fact optimizing the nanostructure matrix is obviously important to observe a reliable switching memory. This report 
implies some interesting features, which may help better understand the mechanism of electrical switching in metal oxide nanostructure polymeric network. Conceivable mechanism for the resistive switching in this system is following with temperature measurement. In this work it is shown that the XRD pattern after switching appears with different crystalline planes, plus temperature dependent studies reveal that conduction of both on and off states is weak thermal activated. Surprisingly, the carrier transport is the same for both on and off-states. Difference between states comes from the dramatic changes in the carrier density. The main mechanism of charge transport for on-state is tunneling. The charge transport leads to SCLC in higher voltages pulse for the off state. The mechanism will be explained based on percolation concepts. A myriad of conducting percolative paths are created after a soft breakdown in oxide. Some of these paths they switch on-and off leading to the observation of RTS noise.

\section{Contribution to Value Creation}

In this work, we utilized the nanostructure silver oxide thin films. Despite of having a sandwich device, the diode is planer in order to explore charge carrier transport within switching phenomena. We reported a memory device, which makes use of thin polymer film hosting a matrix of silver oxide nano structure. With on/off ratio as high as $10^{3}$, a large retention time and good cycle endurance, the nanoparticles based device is a serious candidate to replace currently available non-volatile memories, and is able to improve all the relevant components as a reliable memory device.

In addition, these planar structures have the active layer directly exposed and can be probed by a number of surface analytical techniques like XRD to identify and characterize structural changes that occur in the devices upon resistive switching.

\section{System Description}

The colloidal solution of silver oxide nano structure in PVP was deposited on top of preformed gold microelectrode arrays fabricated on thermal oxidized silicon wafers. The interdigitated gold microelectrode arrays were separated apart $10 \mu \mathrm{m}$ and 10.000 $\mu \mathrm{m}$ long. Prior to electrical measurements the samples were also pumped in vaccum to remove further any residual solvent. Electrical measurements were carried out using a Keithley 487 picoammeter/voltage source in dark conditions, high vacuum. During all the measurement the conductive silicon substrate is kept grounded to prevent charging of the $\mathrm{SiO}_{2}$ layer (see inset of Fig.1(a)).

\subsection{Current-Voltage Characteristics}

Initially, the oxide-polymer matrix is highly insulating and the diodes behave as capacitors. In order to induce bistable resistive states the diode must be submitted to an electric field near to dielectric breakdown. This is known as electroforming and in practice corresponds to a soft-breakdown of the dielectric layer. The details of this breakdown process are still elusive. It is usually assumed that a network of defects is created by the strong electric field. Soft-breakdown is usually distinguished from hard breakdown because the electric power is kept low preventing the propagation of the 
damage in the dielectric by Joule heating. In practice the voltages required to induce soft breakdown are typically $\sim 48 \mathrm{~V}$ for a $10 \mu \mathrm{m}$ channel length between electrodes corresponding to an electric field of $4.8 \mathrm{MVm}^{-1}$.

After forming the device exhibits the bistable current-voltage characteristics presented in Fig.1 and already reported in our previous work [1].
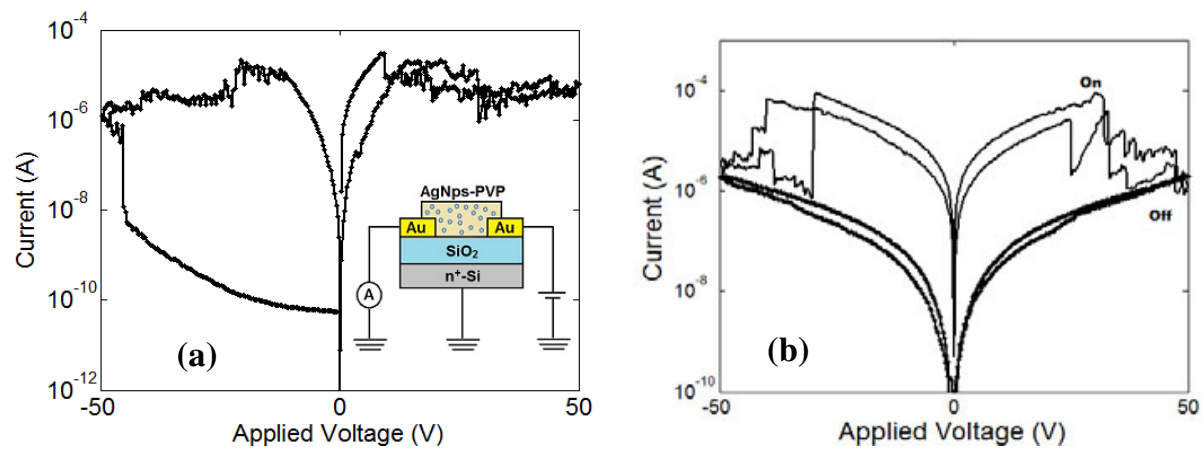

Fig. 1. (a) Electroforming process (inset: device structure). (b). Current-voltage characteristics showing the high conductance on-state and low conductance off-state.

\subsection{Morphological And Structural Characterization}

Silver oxide is a complex multiphase material. Silver being multivalent forms by interacting with oxygen various phases such as $\mathrm{Ag}_{2} \mathrm{O}, \mathrm{AgO}, \mathrm{Ag}_{3} \mathrm{O}_{4}$ and $\mathrm{Ag}_{2} \mathrm{O}_{3}$. The presence of these phases can be identified by using X-ray diffraction (XRD) techniques. Fig. 2 shows a XRD pattern of the diode. In order, to clarify the physical origin of all the different peaks in the spectrum. The XRD was taken for the (a) silicon wafer substrate, (b) for the diode in its pristine state (i.e. right after thin film deposition and (c) in its memory. The first striking observation is that the pristine diode has a crystal structure different from the electroformed diode. This suggests that under the high electric field the silver nanoparticles act as seed and small silver crystals are formed. The peaksin curve (c) of Fig. 2 match with of the presence of the $\mathrm{Ag}, \mathrm{Ag}_{2} \mathrm{O}$ and $\mathrm{AgO}$ phases.

\subsection{Voltage Dependence}

Fig. 3 shows the I-V characteristics (in a log-log scale) of both resistive states for voltages below the NDR region. In the low field region, both the on- and the off-state follow a power law, i. e. $I=\mathrm{A} V^{n}$ where $\mathrm{n}$ is an exponent factor $(n=1$ for perfect Ohmic conduction) and $\mathrm{A}$ is a coefficient which depends on resistance state. At high fields the off-state clearly shows a distinct slope with the value of 2 . Thus the $I-V$ curve of the off-state show two regions of conduction, viz. (i) nearly ohmic at lower bias and (ii) non-ohmic conduction at higher bias. 


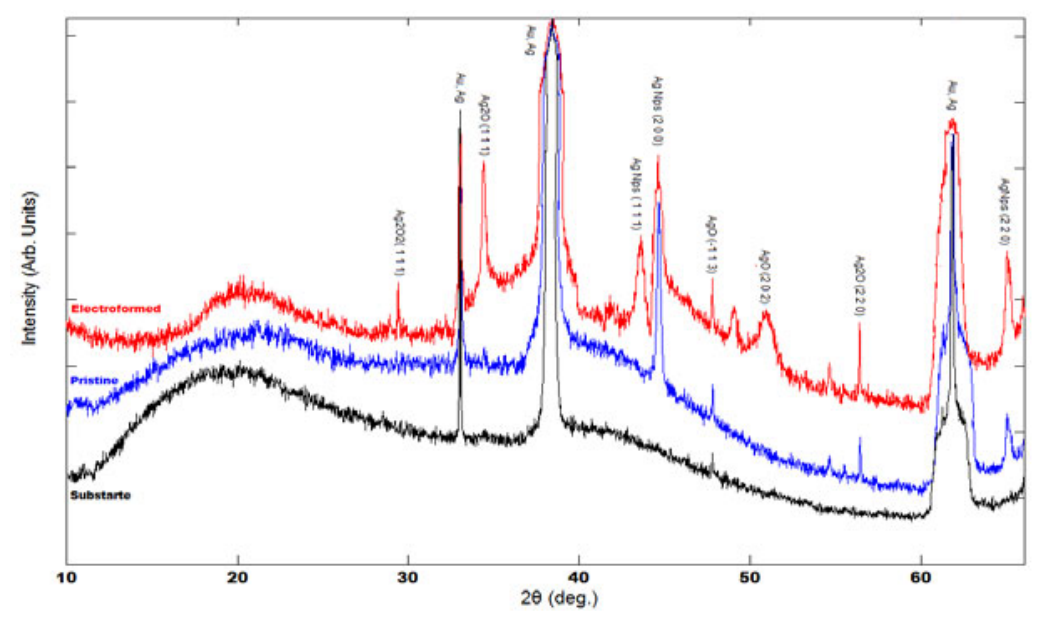

Fig. 2. XRD patterns for the substrate (black), Pristine state (blue), Electroformed state (Red)

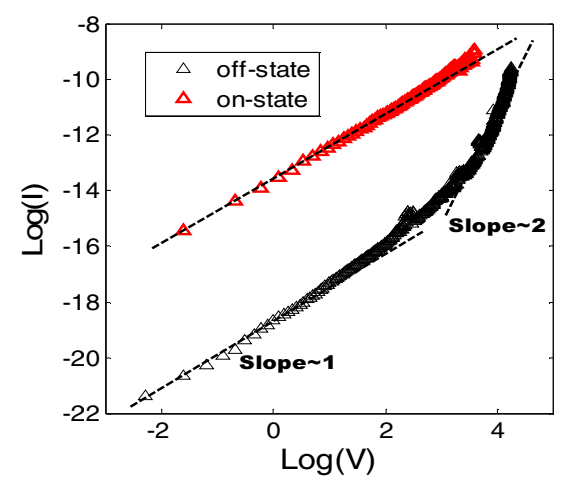

Fig. 3. $\log (\mathrm{I})-\log (\mathrm{V})$ for the on and off-states

For lower bias the injected charge carrier density is lower than the thermally generated carrier density and that leads to ohmic behaviour. At higher bias, as the slope 2 suggests, the conduction in this region is dominated by trap limited space charge limited conduction mechanism [10]. The conduction mechanism in the off-state is then governed by defect states. These states act as carrier trapping centres and after trapping the injected charge from electrodes they become charged and thereby expected to build up a space charge. This buildup of space charge by defects then play a key role in determination of SCLC process. At lower bias up to transition voltage $\left(V_{\mathrm{t}}\right)$, the current density $(J)$ governed by the intrinsic free carrier density $\left(n_{0}\right)$, in the film can be expressed as here $d$ is the gap between the electrodes $n 0$ is the normal volume generated free carrier density and $\mu$ the electron mobility. Using eq. 1 we estimate mobility in the off-state of $\sim 1 \times 10^{-9} \mathrm{~cm}^{2} / \mathrm{Vs}$. 


$$
\mathrm{J}=n o \mu V_{t} / d
$$

\subsection{Temperature Dependence}

To get insight into the charge transport mechanism for both on and off-states we measure the temperature dependence of the current. Fig. 4 shows the Arrhenius plot of the current for an on-state and for two off-states measured with an applied voltage of $3 \mathrm{~V}$. Multiple intermediate off-states are induced by controlling the length of the voltage reset pulse. Longer voltages pulses will induce lower conductance states.

Although, the conduction states are separated by almost 5 orders of magnitude in current, it is striking that the activation energy $(70 \mathrm{meV})$ remains identical. This result shows that upon resistive switching the carrier transport mechanism is not changed but the carrier density increases dramatically.

The low value for the activation energy strongly suggests that the carrier transport is by tunneling or variable ranging hooping between shallow localized states.

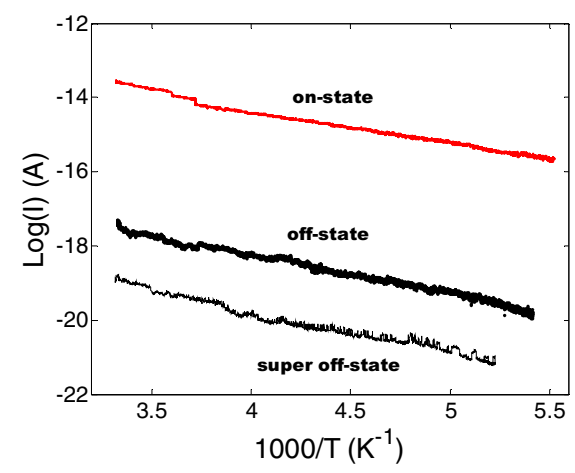

Fig. 4. Arrhenius plot of the current measured at $3 \mathrm{~V}$ for the one state and for two off-states. The activation energy is $70 \mathrm{meV}$ for all the conduction states.

\section{Conclusions}

It is shown that the diode needs an electroforming (soft-breakdown) to become bistable. This process can be considered as a phase-transition from a non-conducting (insulating) to a conducting state. After the soft-breakdown a percolation network of paths is established. The high conductance state is as a result of a myriad of conducting paths within the matrix. For both states the charge transport is via tunneling between nanoclusters, but at the off-state the amount of percolative paths are less than on-state. In fact, due to high electric field some of charge carriers being injected into insulator from nanoclusters where there is no compensating charge present in the insulator. Therefore, they cannot participate in conduction nor have a very small contribution in the conduction. 
Trapped charges in the insulating matrix at higher electric field might lead to a random offset charge disorder. In the presence of such disorder, charge carriers do not flow uniformly through the nanocomposite film. Because of such kind of disorder, it can exist both off and super off states. The $I-V$ curves show nearly Ohmic behaviour at low bias and SCLC at high bias range.

Acknowledgements. We gratefully acknowledge the financial support received from the Dutch Polymer Institute (DPI), project n. ${ }^{\circ}$ 703, from Fundação para Ciência e Tecnologia (FCT) through the research Unit, Center of Electronics Optoelectronics and Telecommunications (CEOT), REEQ/601/EEI/2005 and the POCI 2010, FEDER and the organic chemistry laboratories in Algarve University.

\section{References}

1. Kiazadeh, A., Rocha, P.R., Chen, Q., Gomes, H.L.: Resistive Random Access Memories (RRAMs) Based on Metal Nanoparticles. In: Camarinha-Matos, L.M. (ed.) Technological Innovation for Sustainability. IFIP AICT, vol. 349, pp. 591-595. Springer, Heidelberg (2011)

2. Hickmott, T.W.: Low-Frequency Negative Resistance in Thin Anodic Oxide Films. Journal of Applied Physics 33(9), 2669-2682 (1962)

3. Hickmott, T.W.: Electron Emission, Electroluminescence, and Voltage-Controlled Negative Resistance in Al-AlO2-Au Diodes. Journal of Applied Physics 36(6), 1885-1896 (1965)

4. Barriac, C.: Study of the electrical properties of Al-Al2O3-metal structures. Physica Status Solidi A - Applied Research 34(2), 621-633 (1962)

5. Simmons, J.G., Verderber, R.R.: New Conduction and Reversible Memory Phenomena in Thin Insulating Films. Proceedings of the Royal Society of London. Series A 301, 77-102 (1967)

6. Sutherland, R.R.: Theory for Negative Resistance and Memory Effects in Thin Insulating Films and Its Application to Au-ZnS-Au Devices. Journal of Physics D - Applied Physics 4(3), 468-479 (1971)

7. Ansari, A.A., Qadeer, A.: Memory Switching in Thermally Grown Titanium-Oxide Films. Journal of Physics D -Applied Physics 18(5), 911-917 (1985)

8. Ma, L.P., Liu, J., Pyo, S., Xu, Q.F., Yang, Y.: Nonvolatile electrical bistability of organic/metal-nanocluster/organic system. Applied Physics Letters 82(9), 1419-1421 (2003)

9. Paul, S., Pearson, C., Molloy, A., Cousins, M.A., Green, M., Kolliopoulou, S., Dimitrakis, P., Normand, P., Tsoukalas, D., Petty, M.C.: Langmuir-Blodgett film deposition of metallic nanoparticles and their application to electronic memory structures. Nano Letters 3(4), 533-536 (2003)

10. Silva, H., Gomes, H.L., Pogorelov, Y.G., Stallinga, P., de Leeuw, D.M., Araujo, J.P., Sousa, J.B., Meskers, S.C.J., Kakazei, G., Cardoso, S., Freitas, P.P.: Resistive switching in nano- structured thin films. Applied Physics Letters 94, 1-3 (2009) 